Man and Nature

MAN AND NATURE

L'homme et la nature

L'HOMME ET LA NATURE

\title{
Textes et réécritures de textes : le cas des Fêtes grecques et romaines de Louis Fuzelier
}

\section{David Trott}

Volume 3, 1984

URI : https://id.erudit.org/iderudit/1011826ar

DOI : https://doi.org/10.7202/1011826ar

Aller au sommaire du numéro

Éditeur(s)

Canadian Society for Eighteenth-Century Studies / Société canadienne d'étude du dix-huitième siècle

ISSN

0824-3298 (imprimé)

1927-8810 (numérique)

Découvrir la revue

Citer cet article

Trott, D. (1984). Textes et réécritures de textes : le cas des Fêtes grecques et romaines de Louis Fuzelier. Man and Nature / L'homme et la nature, 3, 77-88. https://doi.org/10.7202/1011826ar

Copyright (c) Canadian Society for Eighteenth-Century Studies / Sociéte canadienne d'étude du dix-huitième siècle, 1984
Ce document est protégé par la loi sur le droit d'auteur. L'utilisation des services d'Érudit (y compris la reproduction) est assujettie à sa politique d'utilisation que vous pouvez consulter en ligne.

https://apropos.erudit.org/fr/usagers/politique-dutilisation/ 


\section{Textes et réécritures de textes: le cas des Fêtes grecques et romaines de Louis Fuzelier}

Le Ballet nouveau des Fêtes Grecques \& Romaines a été représenté pour la première fois sur le Théâtre de l'Opéra le Mardy, 13 Juillet. ${ }^{1}$

C'est ainsi que Le Mercure de juillet 1723 annonça la première représentation du ballet héroïque en trois actes et avec prologue du librettiste Louis Fuzelier. En août de la même année les représentations continuaient, mais non sans quelques changements apportés au spectacle:

Mlee [sic] Prévost a produit une éleve sur le Theatre de l'Opera qui a débuté le Jeudy 26 Aoust dans le troisiéme Acte des Fêtes Grecques \& Romaines; ... On la nomme Mlle Richalet; elle n'a pas encore quinze ans.

L'introduction de cette jeune danseuse dut modifier - ne serait-ce que de façon minime - l'exécution du ballet; le texte écrit fut modifié également, si l'on en croit la suite de l'article du Mercure qui cite une chanson écrite en l'honneur de Mlle Richalet. ${ }^{2}$

La mise en espace par laquelle un texte se métamorphose en voix et en mouvements est un processus propre au théâtre, ainsi que l'est celui par lequel des représentations successives évoluent, jusqu'à entraîner parfois la réécriture du script de départ. Il s'agit d'un passage entre deux 'textes': entre un texte écrit et une réalisation scénique que les théâtrologues dénomment 'texte' aussi; entre telle réalisation et une modification 
ultérieure; enfin, entre les modifications initiées par des acteurs et un texte d'auteur dûment réécrit pour les inscrire. La fréquence des 'passages entre deux textes' occasionnés par Les Fêtes grecques et romaines fut particulièrement élevée, surtout si l'on considère que Fuzelier lui-même fit chaque réécriture. Scénariste, parolier, auteur de préface, co-rédacteur du Mercure et probablement critique de son propre ballet, parodiste curieusement acharné contre lui-même, Fuzelier retravailla son sujet non moins de huit fois. Les Fêtes grecques passèrent par deux ébauches, une édition précédée d'une préface critique, un compte-rendu journalistique faisant état d'une version antérieure du ballet, une parodie non-imprimée et non-représentée intitulée $L a$ Rencontre des opéra, une deuxième parodie intitulée Les Saturnales, et une version abrégée de la deuxième parodie intitulée Le Debris des Saturnales.

Ce mouvement continu d'une forme à une autre caractérisait donc la pratique théâtrale de Louis Fuzelier. Relevant d'une esthétique du flux et de l'indécis, cette pratique préférait le dynamisme au statisme. Là où, par exemple, une pièce de Voltaire aboutissait presque invariablement à l'édition, autorisant un René Pomeau à affirmer que 'L'imprimé constitue au dix-huitième siècle la forme éminente de la communication, ${ }^{\prime 3}$ une pièce de Fuzelier tendait à générer une succession de textes plus ou moins éphémères. On sait que de nombreuses pièces de Fuzelier furent imprimées: ses livrets d'opéra parurent dans la série des oeuvres représentées à l'Académie Royale de Musique et publiées par la famille Ballard; on lui connaît une quarantaine de pièces (écrites par lui seul ou en collaboration) dans Le Théâtre de la foire, ou l'Opéra Comique, ${ }^{4}$ ainsi que plusieurs textes publiés séparément. Il reste cependant une grande part de sa production théâtrale de quelque 237 pièces qui se caractérise, soit par des versions multiples, soit par des versions manuscrites et souvent fragmentaires. ${ }^{5}$ Le texte de son opéra le plus célèbre, Les Indes galantes (1735), fut augmenté par l'entrée intitulée 'Les Sauvages.' Le très populaire Scaramouche pédant, pantomime 'publiée' en 1711, fut la quatrième de cinq versions plus ou moins différentes et dont l'existence est attestée par des procès-verbaux ou des manuscrits inédits.

Or, l'intérêt des œuvres flottantes, 'imparfaites,' ou inachevées se reconnaît de plus en plus depuis que Jean Bellemin-Noël ouvrit il y a dix ans déjà la notion de 'texte' aux presque-textes et aux avant-textes:

Il ... faut ... tâcher d'intégrer à la recherche littéraire tout ce à quoi on voudra conférer le statut de texte, à commencer par les presque-textes que sont les œuvres de littératures dites "marginales" (orales, populaires) - cela est en train de se faire - et en continuant par les avant-textes, où qu'on les trouve et quelque texte qu'ils préparent. ${ }^{6}$ 
Sensible aux périls d'un délire terminologique, j'ajouterais néanmoins après-texte à cette liste pour décrire le produit des réécritures successives que furent les innombrables parodies de Fuzelier et de bon nombre de ses contemporains.

Singulier par son ampleur, le foisonnement de textes associé au cas des Fêtes grecques est pourtant typique d'une autre 'forme éminente de la communication' au dix-huitième siècle, le théâtre. ${ }^{7}$ Mais comment capter ce dynamisme? Le corpus peu connu du théâtre de Fuzelier, et en particulier la série de textes écrits autour des Fêtes grecques, nous permet d'en isoler certaines des composantes fondamentales. Cette série constitue une trace importante d'une pratique théâtrale communiquant le théâtre à la fois comme texte achevé, comme lieu et objet de critique, et comme processus de production. C'est cet aspect de mouvance que je veux poursuivre impossible de l'arrêter de façon définitive - dans les propos que voici.

Parmi les papiers personnels de Fuzelier se trouve une feuille avec l'entête 'Pour le Roy/ les festes Romaines ou de julie. .. .' ${ }^{8}$ Ce document où fourmillent les ratures est à peine une ébauche; les sujets dispersés qui formeront les trois actes du ballet des Fêtes grecques, côtoient une variété d'autres sujets, abandonnés ou utilisés ailleurs. ${ }^{9}$ L'amas de motifs gravite autour du mot 'feste, ${ }^{10}$ contient quelques vers rédigés d'un 'prologue' évoquant une participation active du roi, ${ }^{11}$ et renvoie à des recherches ultérieures. ${ }^{12}$ Avant-texte ouvert, certes, mais qui délimite toutefois les possibilités d'un programme de divertissement royal à venir.

Dans le même dossier se trouve une seconde feuille contenant le résultat des recherches de Fuzelier sur certaines fêtes de l'antiquité, ${ }^{13}$ ainsi que le plan sommaire de son ballet. ${ }^{14} \mathrm{D}^{\prime}$ aspect plus soigné que la première feuille, ce document semble avoir été façonné par les exigences d'une mise en spectacle. Aussi rencontre-t-on plus souvent le mot 'jeux,' qui théâtralise 'fêtes,' et qui suggère une préoccupation avec le mouvement ('jeux' au sens de 'concours,' 'exercices militaires,' 'combats,' 'assauts') et avec les problèmes de la concrétisation ('on y representoit,' 'se representoient,' 'jeux sceniques,' et 'on peut mettre en action...'). Si la première feuille laisse entrevoir l'immensité du fonds culturel dans lequel Fuzelier puisera les motifs de son programme, la seconde révèle une structuration de ce fonds sous l'égide du code d'une éventuelle représentation.

Conçues pour être jouées dans la salle des machines au Palais des Tuileries, ${ }^{15}$ les Fêtes grecques finirent par l'être devant un public 'ordinaire' au Théâtre de l'Opéra. On put suivre le spectacle grâce à un livret imprimé. ${ }^{16}$ Fait relativement exceptionnel pour l'opéra, ce texte était précédé d'un 'avertissement' de quatre pages où Fuzelier anticipait les 
critiques qu'on pourrait éventuellement lui adresser. Contraint de "recycler" son texte, il s'efforçait sans doute d'en justifier l'étrangeté en faisant appel au goût de la nouveauté. ${ }^{17}$ Il se félicitait en outre d'avoir exploité l'histoire plutôt qu'une thématique fictive. ${ }^{18} \mathrm{La}$ singularité de cette incursion de l'historique dans un genre voué au merveilleux sera constamment reprise dans les textes ultérieurs de Fuzelier.

Annoncée dans la préface, la portée novatrice des Fêtes grecques est de nouveau proclamée dans un prologue auto-réflexif qui prépare l'action théâtrale. Clio, Muse de l'histoire, fait savoir à Erato, Muse de la musique, que la vérité a aussi droit de cité dans l'art de l'opéra. ${ }^{19}$ Apollon, qui préside la rencontre des muses, annonce que les jeux d'Erato ont aussi besoin de l'appui de la danse, figurée par Terpsicore. ${ }^{20}$

Le ballet lui-même met en scène trois couples d'amoureux célèbres dont les tête-à-tête ouvrent la voie à trois fêtes dansées. Le premier acte, 'Les Jeux olympiques,' commence par l'entretien d'Alcibiade et Timée, le deuxième, 'Les Baccanales,' par celui de Marc-Antoine et Cléopâtre, et le troisième, 'Les Saturnales,' par celui de Tibulle et Délie. Le spectacle esquissé dans les papiers préliminaires a subi un processus d'apprivoisement; les images vigoureuses de jeux, d'assauts, de combats, ont cédé le terrain à une action nettement plus policée, imposée par les conventions chorégraphiques de l'époque. Le texte d'un spectacle grandiose imaginé pour fêter la majorité de Louis $\mathrm{XV}$ a été réécrit en divertissement d'été bien moins ambitieux.

Le compte-rendu du ballet publié dans Le Mercure de juillet en constitue une autre réécriture. Si Fuzelier ne le fit pas tout entier, tout au moins peut-on voir les traces de sa collaboration dans les trois pages du compte-rendu consacrées à la version 'royale' du texte. ${ }^{21}$ Essentiellement un résumé du prologue et des trois actes joués à l'Opéra, cette analyse mentionne aussi en passant la mise en scène. A deux reprises, elle cite textuellement des phrases de l'avertissement du livret, soulignant dans la première l'originalité d'un sujet de ballet tiré de l'histoire.

En attirant l'attention du lecteur sur une version antérieure des Fêtes grecques, le texte du Mercure accentue l'instabilité des ouvrages de ce genre. En outre, il s'attache au travail d'élaboration par lequel le ballet dut passer avant de devenir texte parachevé. Cette façon de présenter le théâtre comme processus plutôt que comme produit fini se situait dans le contexte des premiers balbutiements d'une critique théâtrale qui n'existait pas encore en tant que discours autonome en 1723. ${ }^{22}$ En l'absence d'un corpus de réflexions sur l'art proprement théâtral (c'est-à-dire, vu de l'angle scénique, et non pas littéraire), la rubrique des 'Spectacles,' créée 
dans Le Mercure de 1721 par Fuzelier et ses collègues, cherchait à produire l'effet de recul critique qui, selon eux, manquait au spectateurs de l'époque:

Il seroit a souhaitter qu'il parut souvent des Dissertations exactes, qui dévelopassent l'art, les beautez, \& les defauts d'une Piece, qu'un Spectateur ne peut pas toujours saisir dans la representation. ... Quoy que le Theâtre François ait de grands avantages sur ceux de nos Voisins, on ne sçauroit nier cependant qu'il n'y ait bien des defauts à corriger, ce qui ne se peut faire qu'en marquant les écueils de ce genre d'écrire. ${ }^{23}$

Marquer de tels écueils ne fut pas que le travail d'une critique théâtrale émergeant dans les journaux. C'était déjà l'un des objectifs principaux des prologues du temps de Fuzelier. Ces textes reflétant d'autres textes les réécrivaient en quelque sorte par anticipation, en proposant aux spectateurs un cadre enchâssant ou un registre détaché, atténuant ou évaluant le plaisir qu'ils allaient éprouver lors des spectacles ainsi présentés. Quant aux spectacles, perçus simultanément sur deux registres, ils devenaient l'expérience d'un flottement entre deux textes dont ni l'un ni l'autre ne pouvait être en fin de compte définitif. Si l'on en croit le prologue des Fêtes grecques, que doublait l'avertissement imprimé, et auquel faissaient écho les pages du compte-rendu consacrées à une autre version du ballet, le déploiement d'un tel appareil dialogique répondait à un besoin profond chez Fuzelier.

La parodie elle aussi entame un dialogue entre deux textes; dialogue que Fuzelier allait alimenter considérablement en écrivant La Rencontre des opéra. ${ }^{24}$ Ce petit acte en treize scènes, constitue en lui-même un exemple frappant du phénomène de la mouvance textuelle. Cette soi-disant 'parodie' des Fêtes grecques, qui est bien davantage une réécriture de son prologue, se mue en dialogue sur un fait divers (la visite anticipée d'une troupe d'opéra italienne), fait divers qui s'élargit à son tour en appréciation de l'apport de la musique populaire, laquelle appréciation s'avère être le prétexte d'une scène de virtuosité vocale exécutée par un acteur habile.

Le décor de La Rencontre des opéra rappelle celui du prologue des Fêtes grecques, tout en le changeant de façon significative. Dans le ballet, la scène se caractérise par son aspect ordonné, monumental, et formel:

Le théâtre représente au fonds le Temple de Memoire orné des Statües des grands Hommes \& d'Inscriptions à leur loüange. ${ }^{25}$

Lieu de la cérémonie publique, cet espace est investi par une troupe de 
danseurs rangés sous les ordres du dieu Apollon. ${ }^{26}$ Par contre, l'espace de La Rencontre quitte la place publique des Fêtes pour l'intimité de l'école d'Erato. L'art (de l'Opéra) cesse d'être vu de l'extérieur pour l'être de l'intérieur; les trois actes des Fêtes grecques sont considérés du dehors comme unités détachées à suivre, le temple et les statues pour leur part symbolisent un 'déjà accompli,' alors que dans La Rencontre la création se voit du dedans comme répétition et composition, un processus en cours et non achevé.

Les différentes séquences de la Rencontre maintiennent le même effet d'irrésolution, soit en renversant ce qui avait déjà été admis dans Les Fêtes grecques, soit en multipliant des moments révélateurs de mises en abyme. La surprise causée par la présence de la Muse de l'histoire dans le prologue des Fêtes grecques fait de Clio une figure d'intruse. L'intrusion, réussie dans le ballet, échoue dans $L a$ Rencontre lorsque l'esclave d'Erato renvoie Clio sous prétexte que la fête des Saturnales dispense les musiciens de travailler. Le départ subit de l'Histoire prive la parodie des sujets du ballet tout en ouvrant la voie à une chasse aux textes pour combler la lacune. Cette chasse devient le prétexte d'un défilé de plusieurs personnages reflétant le théâtre (auteur, spectateur, etc.), chacun reprenant divers éléments des Fêtes grecques.

A titre d'exemple, le premier, un Député des beaux esprits, évoque le ballet sous l'angle de l'écriture de sa parodie. En dépit du scepticisme de Chriseïs, esclave d'Erato, le Député affirme qu'une parodie de ballet est concevable:

LE DEPUTE: Eh! bien pour abreger je crois que Malgré l'aridité de la Matiere la parodie d'un Ballet peut se risquer.

CHRISEIS: Et Moy je vous soustiens qu'il est impossible qu'elle soit Seulement Tolerable. Comment critiquer l'action d'une piece qui n'en a point? d'une piece ou chaque acte est composé de personnages nouveaux qui expedient deux petites scenes pour cedder le Terrain à la Danse?

(sc. 4)

En même temps qu'il se livre à une réflexion sur son propre ballet, Fuzelier se livre à une réflexion sur cette réflexion. Deux scènes plus tard, Erato envisage l'écriture d'une deuxième parodie composée cette fois-ci, dit-on, par l'auteur lui-même des Fêtes grecques:

ERATO: [Chriseïs] pretend nous donner une Parodie composée par L'auteur même de l'ouvrage apostrophé

LE DEPUTE: Le Phenomene est rare!

ERATO: Et n'en sera peut-être pas plus brillant

LE DEPUTE: Eh! pourquoy Diantre cet auteur la a $t$ il la demangeaison de se plai- 
santer luy même? tandis que tout Paris fourmille de poetes charitables qui sont tous prests a luy rendre ce petit service la avec plaisir.

Si La Rencontre des opéra ne vit jamais les feux de la rampe, Les Saturnales en furent illuminées les 2 et 3 septembre 1723. ${ }^{27}$ Vite tombées, elles furent retirées de l'affiche des Comédiens Italiens pour réapparaître trois jours plus tard en un seul acte, sous le titre Le Debris des Saturnales. ${ }^{28}$ Cette réécriture d'une réécriture transfère l'action dans une guinguette où défilent plusieurs personnages des Fêtes grecques. Sous le signe libérateur des Saturnales - et avec l'aide du vin de la guinguette - les actions et les personnages du ballet subissent des dévalorisations et/ou mises en évidence. On dit de Timée, 'Son esprit ne paroît guere ...', de la volte-face d'Antoine, 'Vous aves eté l'amour de Cleopatre aussi promptement que son Juge,' et de Tibulle, 'il a l'esprit un peu trop lourd.' Ces critiques s'accompagnent de clins d'oeil au public, comme au moment où Cléopâtre repousse Marc-Antoine en disant: 'on nous regarde au moins' (sc. 9).

Le groupe de textes relevant des Fêtes grecques et romaines a illustré une pratique de la mouvance, trait on ne peut plus théâtral. D'autre part, les différents documents que nous avons considérés ont livré plusieurs cas d'une tripartition dynamique de l'événement théâtral en texte achevé, en foyer d'analyse critique, et en procès créateur saisi sur le vif. Au niveau de la réception, ce morcellement se traduit en impressions de flottement plus ou moins continu: flottement de motifs à grouper; flottement des conventions de l'opéra; flottement des genres 'opéra' et 'ballet;' flottement du discours critique entre la préface, le prologue, le compte-rendu, et la parodie. ...

Depuis l'ébauche 'Pour le Roy ...' jusqu'au Debris des Saturnales, il a été possible de suivre des transformations successives des Fêtes grecques. L'on peut dire que ces transformations correspondent aux étapes chronologiques impliquées par les termes avant-texte, texte, et aprèstexte. Grâce à un processus de parachèvement de l'écriture, les brouillons de Fuzelier atteignent une clôture précaire de texte au moment de la présentation de son ballet. L'événement que fut la mise-en-scène des Fêtes grecques, le 13 juillet 1723, au théâtre de l'Opéra, déclencha aussitôt une nouvelle série de réécritures: le texte du compte-rendu du Mercure, le manuscrit de La Rencontre des opéra, la parodie des Saturnales, et la compression rapide de cette dernière en un Debris des Saturnales. Autant de documents textuels - existants ou ayant existé - qui marquent les moments d'arrêt d'un mouvement diachronique vers l'avenir, mouvement qui reflète en miniature l'évolution du théâtre à cette époque. 
Mais l'intérêt du cas des Fêtes grecques ne se réduit pas seulement à l'alignement dans le temps d'une série de documents écrits. Presque tous ces textes renferment les éléments d'un discours réflexif et/ou autocritique, précondition nécéssaire de toute évolution, et qui commence par la feuille 'Pour le Roy ...' dont les ratures constitueraient déjà, d'après Bellemin-Noël, un geste d'écriture critique: Toute rature met en cause l'ensemble de l'écriture. ${ }^{29}$ L'avertissement et le prologue des Fêtes grecques se combinent ultérieurement pour renforcer ce registre critique du ballet: tous les deux soulevant la question d'une thématique convenable à l'opéra, ainsi que celle du rôle de la danse dans les productions d'une Académie de Musique. La fonction critique naissante des articles publiés dans Le Mercure n'a pas besoin d'être tirée en longueur ici. Pour ce qui est de la fonction critique des parodies, il est significatif que La Rencontre des opéra l'ait accentuée en choisissant le prologue des Fêtes grecques pour son évaluation du ballet.

L'article du Mercure envisageant l'établissement d'une critique théâtrale soulignait l'importance d'un recul pour saisir les 'défauts d'une Piece, qu'un Spectateur ne peut pas toujours saisir dans la représentation. ${ }^{\prime 30}$ Il laissait supposer un processus à deux temps; celui d'une réception des impressions immédiates de la représentation, et celui, ultérieur, d'une 'relecture' critique. Dans une certaine mesure, les aprèstextes de Fuzelier (tant journalistiques que dramatiques) permettaient une telle relecture. Toutefois, tous les textes considérés ici offrent à leurs lecteurs/spectateurs la possibilité de dépasser une reception trop linéaire, et de faire coïncider les deux opérations. La présence d'un certain nombre d'éléments auto-référentiels déclenche un jeu réciproque de perceptions qui oscillent entre la réception du texte comme produit fini et la réalisation qu'il n'est qu'un procès en cours.

On a pu assigner aux brouillons un statut d'avant-textes grâce à leur clôture relative. Par contre, les renvois à des 'non-réalisés' tels que l'énoncé 'voir au sujet des festes des anciens, ${ }^{31}$ ou 'on peut mettre en action ...' leur donnent un caractère inachevé et ouvert. De même, la dernière phrase, au futur, de l'avertissement des Fêtes grecques, 'on n'apprendra que trop tôt si on s'est égaré,'32 ainsi que l'assemblage par Apollon dans le prologue des ingrédients (chant, histoire, et danse) du spectacle à suivre, ont pour effet de valoriser le dynamisme et non pas la fixité de l'événement.

En fait, la mise en forme que pouvait constituer l'impression / représentation des Fêtes grecques n'était qu'une mise en forme relative. Sans parler des différences fondamentales entre le 'texte' que fut le livret imprimé, et celui que fut sa première réalisation scénique, les citations dans Le Mercure venant d'une version antérieure du ballet, la réécriture 
des paroles de la chanson qui accompagna le début de Mlle Richalet, et surtout, l'addition d'un nouvel acte, 'La Fête de Diane,' à une reprise des Fêtes grecques en 1734, font que le 'texte' du ballet héroïque ne se fixe pas de façon définitive. Il n'est pas étonnant alors que la phase appelée aprèstexte ne suive pas tant Les Fêtes grecques qu'elle l'accompagne dans le temps, au point d'être inextricablement mêlee au processus originel d'écriture. L'avertissement qui précède le livret et le prologue qui annonce le ballet propre ont entamé le discours autoréférentiel avant même que le texte ne soit reçu par le lecteur/spectateur et, par là, rejoignent au-delà du texte les procès d'écriture en cours que renferment le compte-rendu et les parodies.

L'extraordinaire application que Fuzelier mit à réécrire son texte a de quoi étonner. Cependant, notre propos n'est pas de chercher à savoir s'il s'agit d'opportunisme ou de quelque autre projet plus noble. ... Ce qui importe c'est que la série de textes associés au Fêtes grecques offre une illustration segmentée du geste complexe de la production/réception du fait théâtral. Pendant une période de quelques mois à peine, Fuzelier aborda son sujet plusieurs fois, chaque fois d'un point de vue différent. Il fut auteur, critique, spectateur de lui-même. En cela il fut représentatif de son temps, car le dix-huitème siècle français vit s'épanouir une véritable manie du théâtre: les sujets passaient d'une forme à une autre, les spectateurs d'un théâtre à un autre, les practiciens d'un registre à un autre, l'art du spectacle - en quittant la scène pour faire irruption dans la vie passait d'un monde à un autre. La poussée de l'imprimé, à laquelle contribuait Voltaire, et qui relevait par sa tendance vers l'édition 'définitive' d'un certain goût classique de la transparence et de la permanence dans l'expression, fut contrebalancée par la mise au point d'un mode de communication plus ambigu, fait des nuances, des non-dits, et des qualifications d'un langage en évolution constante. L'ampleur attestée par les textes de Fuzelier d'un dynamisme ennemi de formes contraignantes entraîna à son tour l'exigence d'une nouvelle forme capable de fixer, sans pour autant l'immobiliser, ce caractère de mouvance, c'est-à-dire, capable d'être objet (texte) et procès (écriture/réécriture) simultanément.

Si la postérité nous apprend que Louis Fuzelier n'y a pas entièrement réussi, l'exemple du cas des Fêtes grecques et romaines reste néanmoins éclairant pour qui voudrait comprendre le fontionnement de la mouvance, ou de la théâtralité, dans tel texte du théâtre de Marivaux. Accusé de n'avoir fait que des réécritures sans nombre du même sujet, Marivaux semble effectivement s'être répété en puisant régulièrement dans le fonds conventionnel du théâtre italien et en employant deux fois le titre Surprise de l'amour. Cependant, son œuvre est un entrelacement subtil de textes conventionnels, de création originale et d'interrogations 
sur elle-même en tant que théâtre. Là où il faut faire face dans une pièce telle que Les Acteurs de bonne foi à une concentration de tous les types de passage (entre deux 'textes') dans un seul acte, la fragmentation du phénomène intertextuel en huit textes séparés chez Louis Fuzelier facilite l'analyse d'une mouvance autrement insaisissable.

\section{DAVID TROTT \\ Erindale College}

\section{Notes}

1 Le Mercure, juillet 1723, p. 134.

2 'Voici une Parodie faite pour elle sur l'air qu'elle danse, habillée en Flore à la suite de Mlle Prevost' (Le Mercure, septembre 1723, pp. 581-2). Les paroles de la 'parodie' évoquent une danse dans laquelle l'élève se serait efforcée d'imiter les pas de son mentor. Il faut se rappeler qu'en 1723 le terme 'parodie' gardait encore son sens de 'paroles réécrites sur un air connu.'

3 Catalogue des imprimés de la Bibliothèque Nationale, 'Voltaire,' 2 volumes (214-I et 214-II), 1978, p. xii. Pomeau dit que Voltaire est l'auteur ... le plus copieusement représenté au Départment des Imprimés' (p. xi). En effet 'Voltaire a vécu pour écrire, et pour imprimer' (p. xii). Lorsqu'en 1725 il apprend la parution de trois éditions pirates de son Hérode et Mariamne, il en parle dans les termes suivants: 'Je ne sais à cela que de donner ma piece et d'y corriger le plus de choses que je pourai, afin que l'air de la nouveauté soit joint à la correction dont elle avait besoin' (Best. $\mathrm{D} 243,23$ juillet 1725). La répétition de 'corriger' et 'correction' suggère qu'un texte, à l'étape de sa représentation, est imparfait. Cette imperfection peut être attribuée, soit aux erreurs de celui qui copiait les répliques à vive voix, soit à la nature même de la représentation qui, pour réussir, exploite une 'nouveauté' que l'édition ultérieure aura soin d'éliminer ou d'atténuer.

4 Paris: E. Ganeau, 1721-1737, 10 vol.

5 Pour une discussion plus détaillée du très grand corpus des écrits inédits de Fuzelier, voir mon article 'Louis Fuzelier et le théâtre: vers un état présent,' Revue d'Histoire Littéraire de la France, vol. 83, no. 4 (juillet/août 1983), 604-17.

6 Le Texte et l'avant-texte, Collection 'L', Paris: Larousse, 1972, p. 132.

7 Diderot voyait bien le pouvoir communicatif du théâtre. Il s'agit du passage célèbre où Dorval, conscient de l'utilité des théâtres,' rêve de les transformer en églises, et les acteurs, en prédicateurs: 'Ah! mes amis, si nous allons jamais à la Lampedouse fonder ... un petit peuple d'heureux, ce seront là nos prédicateurs; et nous les choisirons, sans doute, selon l'importance de leur ministère. Tous les peuples ont leurs sabbats, et nous aurons les nôtres. Dans ces jours solennels, on représentera une belle tragédie...' (Entretiens sur le Fils naturel, second entretien, Paris: GarnierFlammarion, 1967, p. 52). 
8 ms. Archives Nationales, $\mathrm{AJ}^{13}$ 1035(4), dans une chemise intitulée 'Extraits, fragments ... pouvant servir au plan d'opéras, de ballets... .'

9 Parmi les sujets abandonnés, se trouvent: 'les festes de julie,' 'une feste françoise,' 'une feste ... persane.' Utilisés ailleurs, furent 'les ages' (représentés en 1718), la foire des fées' (en 1722), et 'la boitte [sic] de pandore' (en 1721).

10 Répété treize fois.

11 'Je [Terpsicore, Muse de la danse] scauray mieux que vous amuser sur la scène

Le plus charman des Rois

Il Regarde vos jeux mais il se mesle aux miens.'

12 Des remarques servant d'aide-mémoire conseillent de 'voir l'explication des fables,' ou bien de 'voir au sujet des festes des anciens.'

13 Ayant effectué les recherches envisagées dans la note 12, Fuzelier donne quelques détails sur divers jeux romains et grecs: 'jeux Capitolins,' 'jeux pirriques,' 'jeux istmiens,' etc.

14 Quatre actes sont envisagés: I. 'feste grecque/ les jeux olimpiques ...'; II. 'feste Romaine/ les baccanales ...'; III. 'feste grecque/ les jeux istmiens ...'; IV. 'feste Romaine/ les Saturnales ... .' Le troisième acte sera supprimé par la suite, et le deuxième, combiné avec 'les festes égiptiennes/ antoine et Cleopatre,' ce dernier motif figurant ailleurs sur la feuille.

15 'Ce ballet avoit eté composé pour être representé sur le theatre des Thuilleries. L'auteur animé de l'honneur D'amuser le Roy dans un temps ou il vouloit bien embellir les spectacles, en Daignant s'y meler luy même, avoit imaginé de l'amener dans un Divertissement digne d'un aussi grand roy ...' (Duchaiseau, 'Histoire des théâtres,' ms inédit en 6 cartons, article sur Fuzelier, p. 6 . Voir Bibliothèque de l'Opéra, Rés. 925).

16 A Paris, Chez La Veuve de Pierre Ribou, MDCCXIII.

17 'Les Fêtes Grecques \& Romaines forment un Ballet d'une espece toute nouvelle ...' ou bien, 'On s'est écarté d'une route frayée depuis longtemps ...' ('Avertissement,' pp. iii et vi).

18 A la différence de la plupart des auteurs de ballets qui cherchent l'inspiration 'dans les Champs trop souvent moissonnez de la Mythologie \& du Roman,' Fuzelier se tourne vers l'histoire, plus 'digne d'occuper les génies amateurs du vraisemblable' (ibid. p. iii).

19 La Vérité n'est pas toujours si redoubtable;

L'Histoire aussi bien que la Fable

Peut fournir à vos chants des Héros amoureux.

(prologue)

20 Apolion. - C'est en vain qu'aujourd'hui des chants mélodieux

Sur la Scene appellent les Graces:

Si la Danse n'amuse \& ne charme les yeux

L'Ennui suit les Plaisirs \& vole sur leurs traces.

(prologue)

21 Fuzelier a vraisemblablement écrit tout le compte-rendu. Alors co-rédacteur du 
Mercure avec Dufresney et La Rocque, il a dû répondre lui-même à cet appel que les éditeurs avaient lancé aux auteurs en 1721: 'Comme personne n'est plus capable, generalement parlant, de faire l'Extrait d'une Piéce de Theâtre que celui qui l'a composée, nous avertissons aussi les Auteurs qui doivent craindre qu'un autre ne les fasse pas parler \& penser aussi bien qu'ils croyent avoir fait, de composer eux mêmes les Extraits de leurs Pieces, \& de nous les faire venir ensuite, leur promettant de les insérer dans notre Journal tels que nous recevrons ...' (Mercure, novembre 1721, p. 4).

22 Les Lettres historiques à Mr. $D^{\star \star \star}$ sur la nouvelle comédie italienne (1717-19) de Boindin et les Réflexions critiques (1719) de l'abbé Dubos constituent deux exceptions importantes.

23 Le Mercure, juin et juillet 1721, pp. 131-2.

24 Ms. B.N. f.fr. 9333.

25 Prologue des Fêtes grecques et romaines.

26 'Les Eleves d'Erato s'y trouvent rassemblez par l'ordre d'Apollon pour seconder les desseins de la Muse de l'Histoire' (prologue).

27 Texte disparu. Le manuscrit portant le titre 'Les Saturnales' (ms. B.N. f.fr. 9332) est en réalité 'Le Debris des Saturnales.'

28 Voir Le Debris des Saturnales. ms. B.N. f.fr. 9332. Ce texte se trouve également dans Le Dictionnaire des théâtres de Paris, vol. V, pp. 39-55.

29 Le Texte et l'avant-texte, p. 5.

30 Voir page 81, note 23.

31 Voir note 12.

32 Avertissement des Fêtes grecques, p. vi. 